\section{Portal venous perfusion steal causing graft dysfunction after orthotopic liver transplantation: serial imaging findings in a successfully treated patient}

\author{
Minsu Lee ${ }^{1,2}$, Sang Kyum Kim ${ }^{3}$, Yong Eun Chung ${ }^{1,2}$, Jin-Young Choi ${ }^{1,2}$, Mi-Suk Park ${ }^{1,2}$, \\ Joon Seok Lim ${ }^{1,2}$, Myeong-Jin Kim ${ }^{1,2}$, Honsoul Kim ${ }^{1,2}$ \\ 'Department of Radiology, ${ }^{2}$ Research Institute of Radiological Science, Severance Hospital, \\ Yonsei University College of Medicine, ${ }^{3}$ Department of Pathology, Yonsei University College \\ of Medicine, Seoul, Korea
}

A 53-year-old male with hepatocellular carcinoma underwent orthotopic liver transplantation. Preoperative computed tomography revealed main portal vein luminal narrowing by flat thrombi and the development of cavernous transformation. On post-transplantation day 1, thrombotic portal venous occlusion occurred, and emergency thrombectomy was performed. Subsequent Doppler ultrasonography and contrast-enhanced ultrasonography confirmed the restoration of normal portal venous flow. The next day, however, decreased portal venous velocity was observed via Doppler ultrasonography, and serum liver enzymes and bilirubin levels remained persistently elevated. Direct portography identified massive perfusion steal through prominent splenorenal collateral veins. Stent insertion and balloon angioplasty of the portal vein were performed, and subsequent Doppler ultrasonography demonstrated normalized portal flow parameters. Afterwards, the serum liver enzymes and bilirubin levels rapidly normalized.

Keywords: Liver transplantation; Portosystemic shunt, surgical; Ultrasonography, Doppler; Perfusion

\section{Introduction}

After liver transplantation, imaging studies focus mainly on the prompt assessment of anastomosis complications that might threaten luminal patency. Certain conditions can compromise graft perfusion despite patent hepatic vasculatures, however, leading to devastating graft dysfunction and/ or failure. Long-standing portal hypertension leads to the development of extensive portosystemic collateral vessels including spontaneous splenorenal shunts [1]. The shunts can serve as channels that significantly divert the portal venous flow, exhausting the normal hepatopetal perfusion. The potential of such stealing of portal perfusion is well acknowledged in the surgical literature [2-4] but receives scant attention in the field of radiology, and few articles focus on the imaging of this disease category [5]. We describe the findings of serial imaging studies including Doppler ultrasonography,

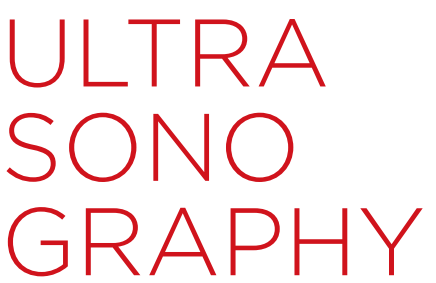

\section{CASE REPORT}

http://dx.doi.org/10.14366/usg. 15012 pISSN: 2288-5919 • elSSN: 2288-5943 Ultrasonography 2016:35:78-82

Received: February 7, 2015 Accepted: February 24, 2015

Correspondence to: Honsoul Kim, MD, Department of Radiology, Yonsei University College of Medicine, 50-1 Yonsei-ro, Seodaemungu, Seoul 03722, Korea

Tel. $+82-2-2228-7400$

Fax. +82-2-393-3035

E-mail: pine0205@yuhs.ac

This is an Open Access article distributed under the terms of the Creative Commons Attribution NonCommercial License (http://creativecommons.org/ licenses/by-nc/3.0/) which permits unrestricted noncommercial use, distribution, and reproduction in any medium, provided the original work is properly cited.

Copyright (C) 2016 Korean Society of Ultrasound in Medicine (KSUM)

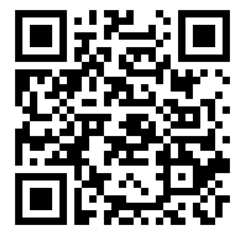

How to cite this article:

Lee M, Kim SK, Chung YE, Choi JY, Park MS, Lim JS, et al. Portal venous perfusion steal causing graft dysfunction after orthotopic liver transplantation: serial imaging findings in a successfully treated patient. Ultrasonography. 2016 Jan;35(1):78-82. 
contrast-enhanced ultrasonography, computed tomography (CT), and conventional angiography (direct portography) of a patient who was successfully treated for massive portal perfusion steal after orthotopic liver transplantation. This study was approved by the Institutional Review Board of our institution, and a waiver of informed consent was obtained.

\section{Case Report}

A 53-year-old male with underlying chronic viral hepatitis B and hepatocellular carcinoma underwent orthotopic liver transplantation. Preoperative CT revealed partial luminal narrowing of the extrahepatic portal vein by chronic flat thrombi and the development of cavernous transformation along with large splenorenal collateral vessels (Fig. 1A, B). The preoperative serum aspartate aminotransferase (AST), alanine transaminase (ALT), and bilirubin levels were $34 \mathrm{IU} / \mathrm{L}, 15 \mathrm{IU} / \mathrm{L}$, and $1.6 \mathrm{mg} / \mathrm{dL}$, respectively. Although with technical difficulty due to the thrombi, end-to-end anastomosis of the portal vein was successfully conducted. Ligation of the large splenorenal shunt was considered during the operation but was not attempted, because the surgeon judged the technical difficulty and risk of potential vascular rupture during ligation to be overwhelming.

Routine Doppler ultrasonography on postoperative day 1 revealed very weak blood flow signals in the portal vein and the hepatic vein. Contrast-enhanced CT was immediately performed and identified a small thrombus at the anastomosis site causing portal vein occlusion. The serum AST, ALT, and bilirubin levels were 2,405 IU/L, $1,433 \mathrm{IU} / \mathrm{L}$, and $4.3 \mathrm{mg} / \mathrm{dL}$, respectively. An emergency operation was performed, and the portal vein thrombus was removed. A few hours later, a Doppler ultrasonography study using a GE Logiq E9 Ultrasound System (GE Healthcare, Milwaukee, WI, USA) with a 1-5$\mathrm{MHz}$ convex probe demonstrated intact hepatic vascular perfusion, and the portal vein showed an intact color Doppler signal with a flow velocity of approximately $20-30 \mathrm{~cm} / \mathrm{sec}$ (Fig. 1C). Contrastenhanced ultrasonography was performed after bolus injection of $2.4 \mathrm{~mL}$ SonoVue (Bracco S.p.A., Milan, Italy) through a 21-gauge peripheral intravenous cannula followed by $5-\mathrm{mL}$ saline flush and a robust hepatopetal influx of intravenous contrast agent, confirming the patency of both the hepatic artery and the portal vein (Fig. 1D). The serum AST, ALT, and bilirubin levels at that point were 4,727 IU/L, 4,357 IU/L, and $6.9 \mathrm{mg} / \mathrm{dL}$, respectively.

The next day, routine Doppler ultrasonography using an Acuson X300 System (Siemens Medical Solutions, Erlangen, Germany) with a 2-5-MHz convex probe suggested compromised portal perfusion, as the portal venous flow velocity sampled near the liver hilum was around $10 \mathrm{~cm} / \mathrm{sec}$ (Fig. 1E). Moreover, power Doppler ultrasonography identified weak intermittent portal venous flow signals at the graft periphery, which did not seem to properly propagate within the vessel lumen (Fig. 1F). Meanwhile, the hepatic arterial color Doppler signal and spectral wave pattern were normal. Serum chemistry levels were persistently elevated (AST, $955 \mathrm{IU} / \mathrm{L}$; ALT, 2,698 IU/L; bilirubin, $10.4 \mathrm{mg} / \mathrm{dL}$ ).

Subsequent $\mathrm{CT}$ was immediately performed to exclude recurrent portal vein thrombosis and related vascular occlusion, but the portal vein appeared patent. The scan revealed partial luminal narrowing in the pre-anastomosed recipient segment with a diameter of 0.6 $\mathrm{cm}$ (Fig. 1G), which was apparently irrelevant to the anastomosis complication, because such narrowing was also observed in a preoperative CT scan without remarkable interval change (Fig. 1B). Liver parenchymal ischemia was suspected because multifocal wedge-shaped regions of decreased perfusion in the portal venous phase were observed. At that point, the presence of a large splenorenal shunt gained our attention. Suspecting portal venous perfusion steal, the patient underwent direct portography, which confirmed massive diversion of the portal venous circulation through the splenorenal shunt (Fig. 1H). The pressure gradient across the narrowed segment was $3 \mathrm{~mm} \mathrm{Hg}$. Prompt ablation of the splenorenal shunt was not possible. Instead, vascular stent insertion and balloon angioplasty were performed to lower the hepatopetal portal flow resistance and thus the fraction of perfusion steal. After the intervention, Doppler ultrasonography showed restoration of the portal venous flow (Fig. 11), and the blood chemistry including the liver enzymes and bilirubin levels rapidly normalized. A CT scan performed one month later showed full expansion of the portal venous stent with excellent portal perfusion. Although a large splenorenal shunt remained, the liver parenchyma recovered a normal appearance (Fig. 1J). The patient did not experience additional adverse events, and the liver graft maintained excellent function for the next two and a half years until the present.

\section{Discussion}

We described the imaging findings of a transplantation patient who experienced a failing liver graft caused by a portal flow steal phenomenon. Preoperative $\mathrm{CT}$ revealed the presence of risk factors previously described in the literature, such as a portosystemic shunt with a large diameter and portal vein stricture/thrombosis [6]. Serial post-transplantation Doppler ultrasonography revealed fluctuating Doppler parameters including portal venous flow velocity and color flow signal, which we interpreted to reflect perfusion vulnerability.

Patients who receive orthotopic liver transplantation are at risk of portal vein thrombosis with an incidence ranging between $2.1 \%$ and $13 \%$ [7]. Our patient suffered from acute portal vein 


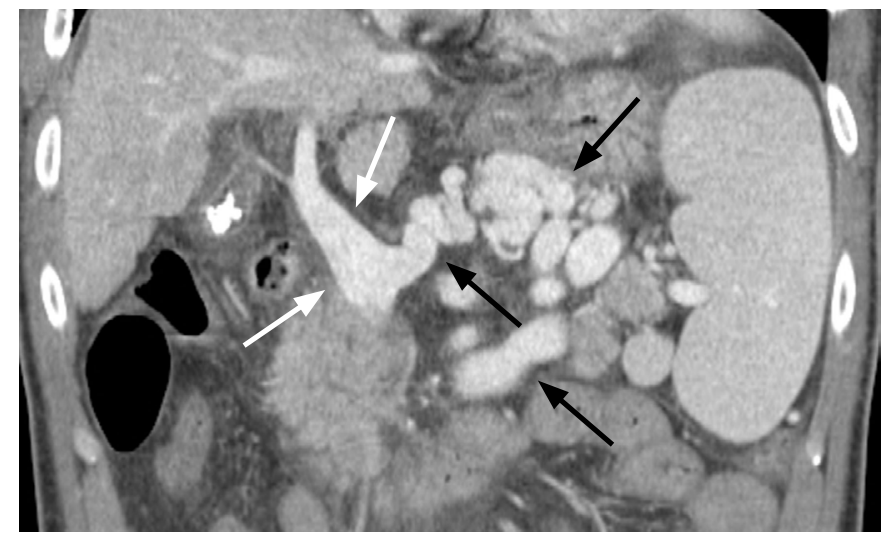

A

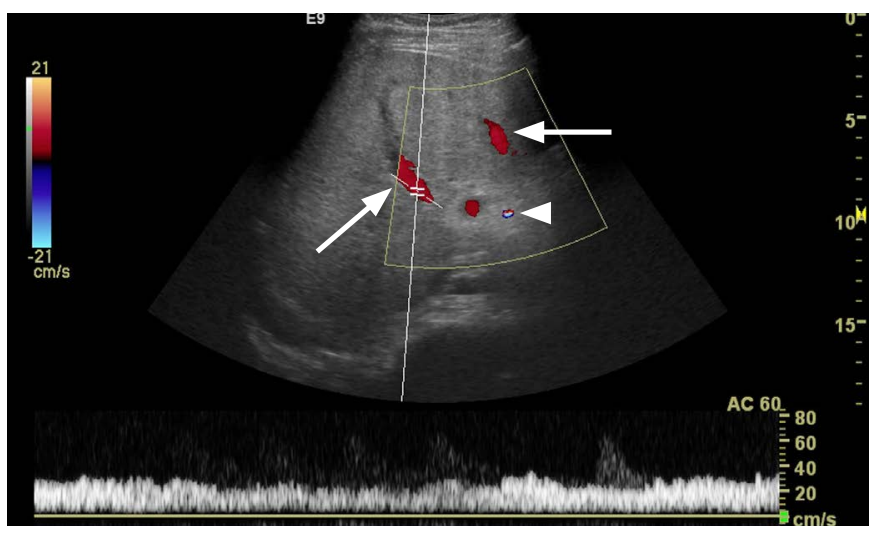

C

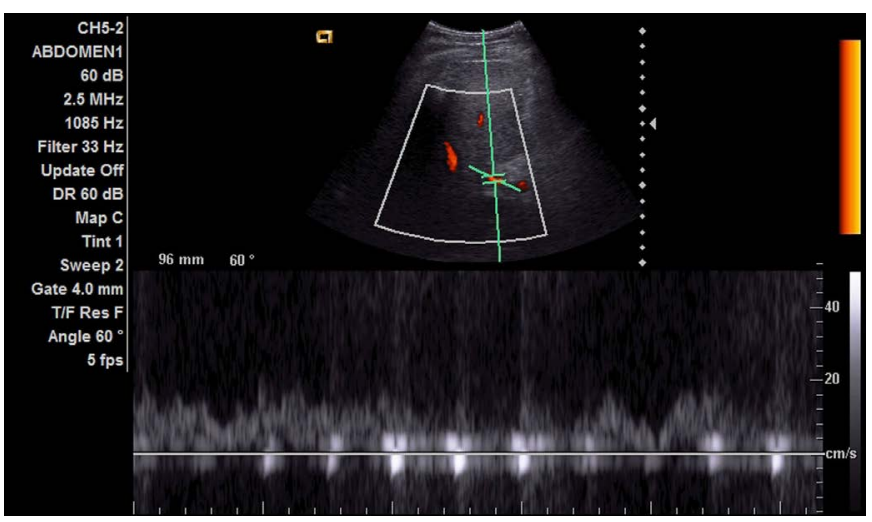

$\mathrm{E}$

Fig. 1. A 53-year-old male who underwent orthotopic liver transplantation.

A, B. Coronal reconstructed image of portal venous phase images of a computed tomography (CT) scan obtained 6 years (A) and 2 weeks (B) before transplantation. Compared with the older image, the image obtained 2 weeks prior to surgery demonstrates the development of cavernous transformation (white arrowhead) and partial luminal narrowing of the extrahepatic portal vein (white arrows) due to a chronic flat thrombus (black arrowhead). Large splenorenal collateral vessels (black arrows) are observed in both studies. C, D. On Doppler ultrasonography $(C)$ performed on post-transplantation day 2 a few hours after emergency portal venous thrombectomy, the flow velocity sampled at the right portal vein was $20-30 \mathrm{~cm} / \mathrm{sec}$. An unequivocal hepatopetal color signal in the portal vein branches (arrows) and the right hepatic artery (arrowhead) is seen. Subsequent SonoVue contrast-enhanced ultrasonography (D) confirms unequivocally the influx of the intravenous contrast agent in the right portal vein (arrows) and hepatic artery (arrowheads). E, F. Doppler ultrasonography delivered on post-transplantation day 3 shows a continuous flow signal in the portal vein near the hilum (E), but even there the flow velocity had fallen to approximately $10 \mathrm{~cm} / \mathrm{sec}$. At the periphery of the graft (F), the power Doppler flow signal (arrowhead) looks weak and only manages to partially propagate within the portal vein lumen (arrows). 


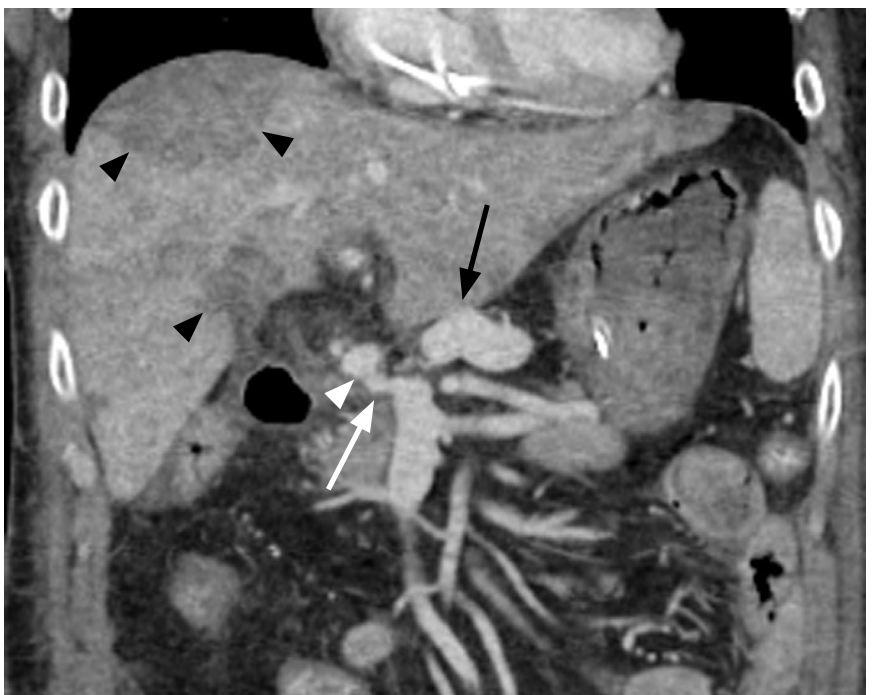

G

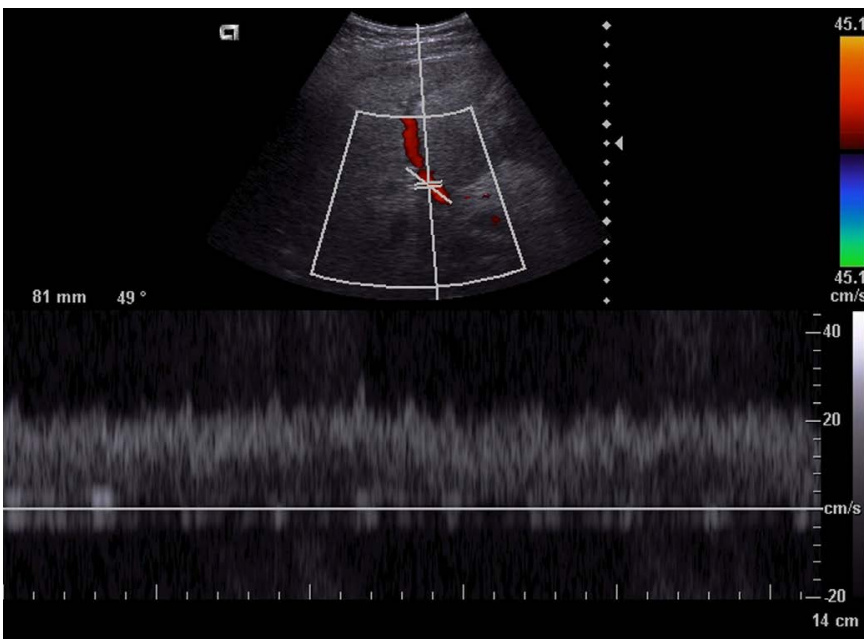

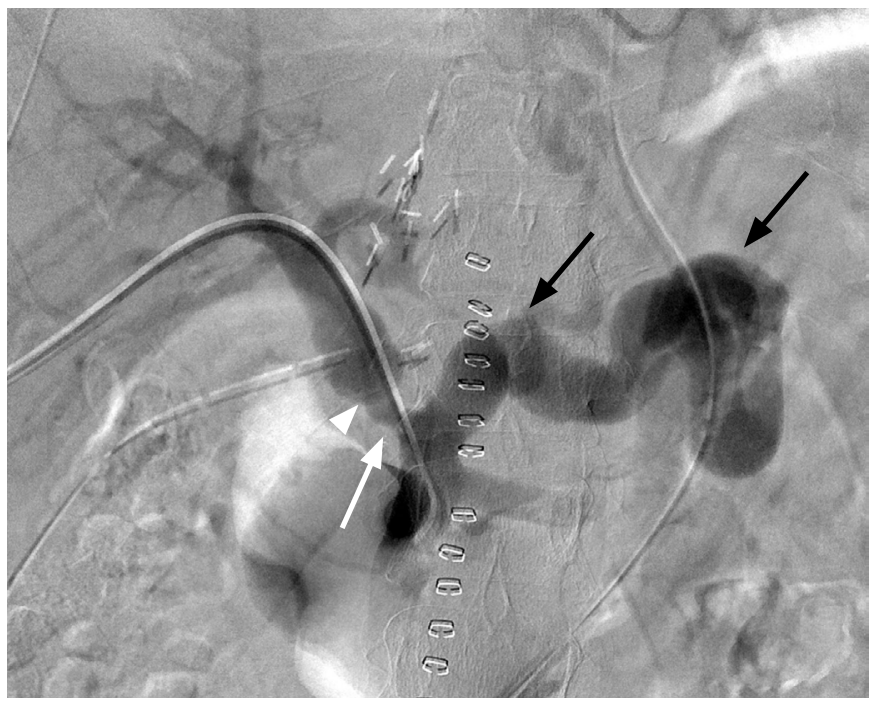

$\mathrm{H}$

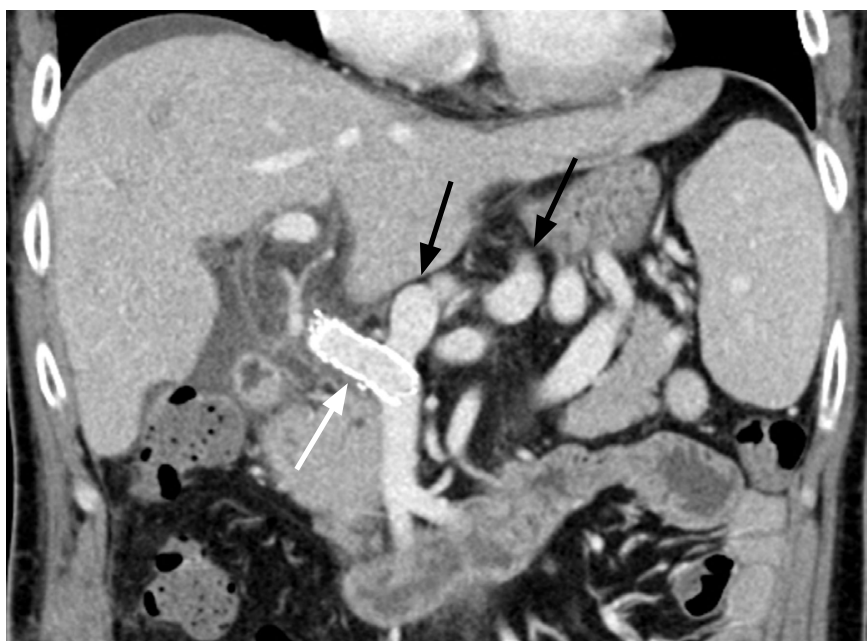

J

Fig. 1. G. Coronal reconstructed image of the portal venous phase of CT shows that the portal vein (white arrow) is patent. Luminal narrowing of the recipient-side extrahepatic portal vein (white arrow) is present with a diameter of $6 \mathrm{~mm}$ but was not considered a complication of the anastomosis (white arrowhead) because the same narrowing was observed on preoperative CT (B). Persistent splenorenal shunts are present (black arrow). The graft liver shows multifocal wedge-shaped parenchymal regions with decreased perfusion, suggesting ischemic injuries (black arrowheads). H. Direct portography demonstrates marked portal perfusion steal into the splenorenal shunt (black arrows). The partially narrowed recipient portal vein (white arrow) and the diameter mismatch at the anastomosis site (white arrowhead) are displayed. The pressure gradient across the narrowed segment was $3 \mathrm{~mm} \mathrm{Hg}$. Vascular stent insertion and balloon angioplasty were immediately performed. I. Doppler ultrasonography after portal vein intervention reveals restoration of the portal venous color flow signals, including those located at the graft periphery. The flow velocity sampled at the right portal vein measured $20 \mathrm{~cm} / \mathrm{sec}$. J. A coronal reconstruction image from CT obtained 1 month after liver transplantation shows the stent (white arrow) remaining fully expanded and patent. Splenorenal collateral vessels persist (black arrows).

thrombi detected on postoperative day 1, which were removed by emergency thrombectomy surgery. Afterwards, our main concern was the early detection and exclusion of potential recurrence of portal vein thrombosis, which led us to perform contrast-enhanced ultrasonography in addition to routine Doppler ultrasonography.
We determined the portal vein to be patent, as we observed the robust influx of the contrast agent through the portal vein. One day later, Doppler ultrasonography findings suggested compromised portal venous perfusion; however, no vascular occlusion event was identified by subsequent CT. 
If a large portosystemic shunt persists after liver transplantation, portal venous perfusion can become significantly compromised, especially if the diameter of the collateral vessel is larger than that of the portal vein. Under such conditions, any factor that raises the intrahepatic portal resistance, including but not limited to acute rejection, graft ischemic damage, and vascular complications, can trigger meaningful diversion of the portal flow $[3,8]$. Consequently, a portal flow steal phenomenon can be aggravated, resulting in graft dysfunction or even failure despite the vessels being patent $[6,9]$, which we assume was the case for our patient.

Our patient demonstrated a narrowed extrahepatic portal vein caliber in the preoperative period. A short segmental luminal narrowing remained at the recipient portion of the portal vein, but the degree of narrowing seemed similar to that identified by preoperative $\mathrm{CT}$, indicating that significant anastomosis complication did not occur. We believe, however, that the prominent cavernous transformation observed prior to transplantation was probably an important factor that was initially overlooked. The collateral vessels were ligated during the transplantation operation, and consequently, a relatively large portion of the effective hepatopetal portal flow channels became deprived, inevitably increasing the portal venous pressure, which we believe contributed to triggering and/or aggravating the portal perfusion steal. The failing Doppler ultrasonography parameters as well as the failing liver function were rapidly restored after balloon angioplasty and stent insertion, although it is worth noting that the large splenorenal shunt remained.

In conclusion, radiologists should be aware of the potential risks of portosystemic steal phenomena, which can develop in the absence of vascular anastomosis complications but still lead to graft dysfunction. Preoperative imaging studies should be optimized and carefully reviewed to detect risk factors such as portosystemic collaterals, thrombi, and cavernous transformation. Upon Doppler ultrasonography, portal flow parameters can fluctuate or rapidly deteriorate despite patent vessels, in which case a portal steal phenomenon should be suspected.
ORCID: Minsu Lee: http://orcid.org/0000-0003-1943-3677; Sang Kyum Kim: http://orcid.org/0000-0003-0768-9923; Yong Eun Chung: http://orcid.org/00000003-0811-9578; Jin-Young Choi: http://orcid.org/0000-0002-9025-6274; Mi-Suk Park: http://orcid.org/0000-0001-5817-2444; Joon Seok Lim: http://orcid.org/00000002-0334-5042; Myeong-Jin Kim: http://orcid.org/0000-0001-7949-5402; Honsoul Kim: http://orcid.org/0000-0002-2367-234X

\section{Conflict of Interest}

No potential conflict of interest relevant to this article was reported.

\section{References}

1. De Carlis L, Del Favero E, Rondinara G, Belli LS, Sansalone CV, Zani B, et al. The role of spontaneous portosystemic shunts in the course of orthotopic liver transplantation. Transpl Int 1992;5:9-14.

2. Castillo-Suescun F, Oniscu GC, Hidalgo E. Hemodynamic consequences of spontaneous splenorenal shunts in deceased donor liver transplantation. Liver Transpl 2011;17:891-895.

3. Ploeg RJ, D'Alessandro AM, Stegall MD, Wojtowycz M, Sproat IA, Knechtle SJ, et al. Effect of surgical and spontaneous portasystemic shunts on liver transplantation. Transplant Proc 1993;25:19461948.

4. Lee SG, Moon DB, Ahn CS, Kim KH, Hwang S, Park KM, et al. Ligation of left renal vein for large spontaneous splenorenal shunt to prevent portal flow steal in adult living donor liver transplantation. Transpl Int 2007;20:45-50.

5. Horrow MM, Phares MA, Viswanadhan N, Zaki R, Araya V, Ortiz J. Vascular steal of the portal vein after orthotopic liver transplant: intraoperative sonographic diagnosis. J Ultrasound Med 2010;29:125-128.

6. Sanada Y, Mizuta K, Urahashi T, Wakiya T, Ihara Y, Okada N, et al. Impact of posttransplant portosystemic shunts on liver transplantation. World J Surg 2012;36:2449-2454.

7. Woo DH, Laberge JM, Gordon RL, Wilson MW, Kerlan RK Jr. Management of portal venous complications after liver transplantation. Tech Vasc Interv Radiol 2007;10:233-239.

8. Sadamori H, Yagi T, Matsukawa H, Matsuda H, Shinoura S, Umeda $Y$, et al. The outcome of living donor liver transplantation with prior spontaneous large portasystemic shunts. Transpl Int 2008;21:156162.

9. Sekido H, Matsuo K, Takeda K, Morioka D, Kubota T, Tanaka K, et al. Severe fatty change of the graft liver caused by a portosystemic shunt of mesenteric varices. Transpl Int 2002;15:259-262. 